\title{
The Key Technology in Digital Preservation and Communication for Re-gong Thangka
}

\author{
FAN Rong ${ }^{1, a}$ \\ ${ }^{1}$ Shanghai University of Engineering and Science, Shanghai 200000,China \\ âFanRong923@yeah.net
}

Keywords: Re-gong, Thangka, intangible cultural heritage, digital preservation and communication

\begin{abstract}
With the era coming of digitization and informatization, it is necessary to modernize Chinese folk cultures which show distinct ethnic features, such as Re-gong Tangka. Therefore, the establishing of framework, based on the internet, of integrated digitization in intangible cultural heritage is demanded. Meanwhile, corresponding database should be stored for the scientific use of analysis and preservation. In this paper, the main effect and application of digitization in digital preservation and communication for Re-gong Thangka in Qinghai, China through experimental method and comparative research analysis method is got.
\end{abstract}

\section{Background}

Re-gong art dates back to Yuan dynasty, originating from Longwu River bank in Tongren County, the Huangnan Tibetan autonomic prefecture. It is a very important part of art of Tibetan Buddhism in China. Re-gong art, as a typical representative in Chinese civilization, has been formed in a long period of time.

The core of Re-gong art lies in religious culture. Religious culture in Re-gong fuses elements form Chinese Buddhism, Tibetan Buddihsm, Shamanism, Taoism, Islam, and Confucianism, which has developed into a unique religious and cultural landscape in that area. Re-gong art is certainly a magnificent part of Chinese cultural treasure house. In addition, the amazing Re-gong Thangka art attracts most.

Thangka is a traditional way of religious scroll painting, which is to be mounted by silk and hung on the wall for worship. It can be dated back to 10th century AD, and it is precious in terms of history, art, and economy. Thangka has absorbed abundant element of folk painting from minorities, and its creation model, specification requirements and painting language are summed up into systematic painting theories, being inherited through monastic education.

Thangka is usually used to promote religious doctrine, decorate temples and for followers to worship. The fundamental materials are mainly cloth, silk and paper for colored painting. Due to its verisimilitude, gorgeousness, distinct ethnic features, strong color in religion and unique style, it has always been treasured my collectors.

Thangka is commonly used in the propaganda of religious teachings and decorative Temple and believers to accumulate merit. The picture content covers the various sects of Tibetan Buddhism in the deity, Buddhism and Bodhisattvas, Venerable Master, and Tibetan social history, astronomy and geography, Tibetan medicine and pharmacology, historical figures and auspicious patterns etc.. Most of the material is painted Thangka with cloth, silk material and paper material for the bottom, there are many embroidery, normally fabric. Thangka image lifelike, the color is gorgeous, with national characteristics, the distinctive strong religious color and unique artistic style, has always been regarded as treasures collector. Thangka painting art is one of the most representative of the tibetan. The performance of a wide range of subjects, beyond religion, also includes a large number of history and folk custom content. In recent years, Thangka collection has attracted more and more attention of collectors, and record high prices in the auction market. Thangka art spread scope gradually expanded, the number of Thangka in growing, but the old Thangka becomes scarce, especially the age-old boutique is very rare, but the high price to the price level. Minzu University of China professor Wang Jianmin organized the investigation on Tibet Thangka inheritance, and on 
this basis, apply for the national philosophy and Social Science Fund Project "investigation and study of Tibet Thangka heritage status in Tibet". The study on contemporary inheritance of Tibet Thangka as research key, the research emphasizes the subjectivity of Tangka artists, especially pay attention to inspecting family, village, school, temples and other different inheritance of Thangka scene heritage status, mode of philosophy, logic, rules and behavior outstanding engaged in art practice Thangka painters exhibit in response to different the scene process, so as to understand the inheritance mechanism of Tibet Thangka in contemporary society.

\section{Situations and research ideas}

Nowadays, Thangka has been increasingly directed to marketization, from being what is religiously sacred to a piece of artwork and even some commodity purely for profit. As the Internet popularized rapidly, Thangka gradually find its way there in ordinary families. But its original intention to highlight the sacred religious connotation and itself a good artwork are ignored as time goes by. Fewer and fewer young people could totally engage themselves into the study of Thangka painting and its religious connotation since the death of those old craftsmen and the flourished tourism as well as the impact of market economy consciousness, which would gradually bring a grave to Thangka. Therefore, it is quite necessary to protect and inherit Re-gong Thangka through systematic digital techniques.

In recent years, with the spread of national protection of intangible cultural heritage, it has been one of the main contents of Chinese Ethnic and Folk Culture Protection Project to truly, systematically and wholly record those non-material culture heritage which are precious and endangered by means of modern technology like words, recordings, videos and digital multimedia etc. and then build archives and database.

As one of the intangible cultural heritage of the Re-gong Tangka art has been at home and abroad, the current domestic relevant research to the Re-gong Art - Thangka is limited to a situation, resource information and so on of Thangka art form in itself some of the revolutionary era, about how the environment in the current digital media to the core "Thangka of Re-gong Tangka" and protection the inheritance of effective play, did not put forward a system solution. International Thangka collection is, there are many Thangka briefing books publication, but also the lack of system using advanced digital technology for the protection and inheritance of traditional Re-gong Tangka art precedent.

Likewise, we can record the painting techniques of Thangka and the process of teaching by those masters through means mentioned above.

Also, we can make some relevant animations, games and cartoon products, with the help of digital multimedia, to spread its oriental cultural connotation all over the world.

As is followed in figure 1, utilize digital technology in the academic classification of Re-gong Tangka art, cultural information storage and the establishment of symbol database and index database data. Then, utilize the virtual reality technology, the traditional production method of re Gong Thangka painting usage mode, consumption mode, circulation mode, transmission mode of reproduction mode of existence.

This data has increased up 34\% than before the experiment.

The effectiveness of the digital system is expressed as:

$x / y=z \%$

$\mathrm{X}$ means that the number of visitors who use the system. And $\mathrm{y}$ is referred total number of visitors, $\mathrm{z}$ referred as effectiveness.

Among it,

$\mathrm{z}$ is 80 . 


\begin{tabular}{|c|c|c|}
\hline \multirow{4}{*}{$\begin{array}{l}\text { Digital } \\
\text { protection }\end{array}$} & The First Period & The Second Period \\
\hline & \multirow{2}{*}{$\begin{array}{l}\text { The use of digital technology to establish } \\
\text { academic classification, data storage, } \\
\text { information symbol database and } \\
\text { material database }\end{array}$} & Digital reduction and preservation \\
\hline & & The famous image recording \\
\hline & $\begin{array}{l}\text { Knowledge popularization Education Of } \\
\text { Thangka }\end{array}$ & \\
\hline \multirow{5}{*}{$\begin{array}{l}\text { The digital } \\
\text { transmission }\end{array}$} & \multicolumn{2}{|c|}{ The old Tangka paintings digital reduction and preservation } \\
\hline & \multicolumn{2}{|l|}{ Re Gong Thangka artists image recording } \\
\hline & \multicolumn{2}{|l|}{ Digital teaching platform painting techniques } \\
\hline & \multicolumn{2}{|c|}{ Mobile terminal interaction micro-blog material collection } \\
\hline & \multicolumn{2}{|l|}{ The digital variant } \\
\hline
\end{tabular}

Figure 1: The content of the digitization in Thangka

\section{Some digital preservation and communication technology research for Re-gong Thangka}

\section{Digital protection based on reverse engineering}

Reverse engineering refers to the relevant technology from real samples and new products manufacturing. In view of the complex irregular handmade folk art, the use of artificial modeling precision is not high, according to people's modeling, the deviation from the original scale is different, so the use of artificial methods of 3D digital modeling efficiency is low, 3D effect is very difficult to achieve the standard model. In order to effectively quality implementation of 3D digital workflow, complex irregular shapes using reverse engineering scheme, as shown in figure 2. Reverse engineering can be divided into the key technology of data acquisition, data processing, and surface reconstruction.

Data acquisition. Data acquisition is the first step in reverse engineering CAD modeling. According to the different measurement methods, data collection methods are divided into two categories: contact and non-contact measurement, including data acquisition devices typically have three coordinate measuring machine, optical scanner and CT scanner, etc. The quality of data acquisition directly affects the accuracy and completeness of the physical description of the influence of CAD reconstruction, surface and solid models, and quality and therefore, must be 3D data scanner according to the different requirements of different.

Data processing. The key technology of data processing, including multilayer segmentation, noise removing, data reduction, and grid data, etc... First, collecting data from multiple angles and the common coordinate system needs, in order to get the data integration model; secondly, also need to be deleted due to environmental changes and other factors of noise; again is a high data density, such as optical scanning equipment, need to simplify redundant data. Data block also need the reason to collect the physical topology or other collection to fill a vacancy.

Surface reconstruction. The material form of surface scanning and data processing is usually composed of a plurality of surface. Therefore, must first according to the geometric characteristics of point cloud data segmentation. And then a separate for each surface fitting, through the transition surface, crossroads, cutting, rounding, multiple surface are combined into a whole, namely CAD model reconstruction. 


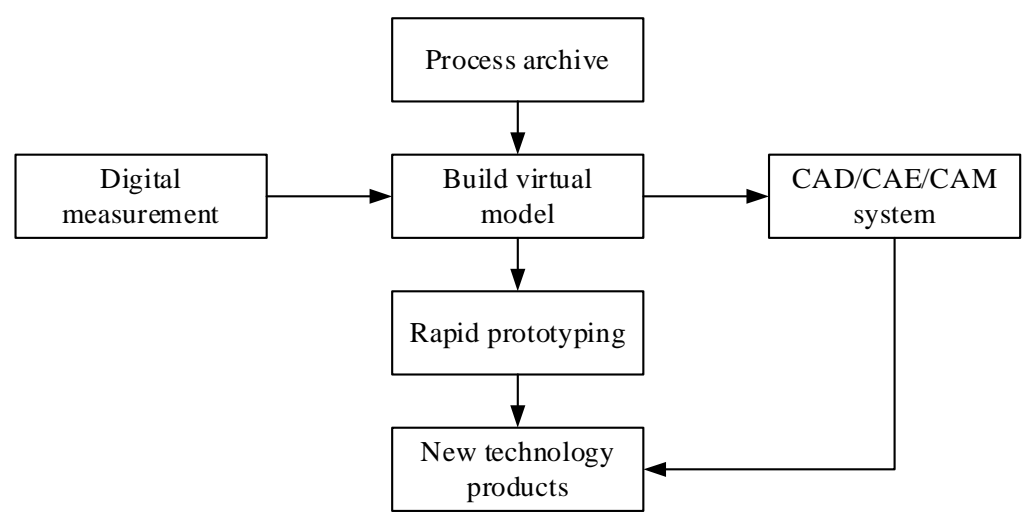

Figure 2.The digital protection technology based on reverse engineering

\section{Folk craft digital protection based on motion capture}

With computer hardware and software technology's fast development and the improvement of the animation, motion capture technology applications include video production, game, virtual reality, human engineering research, simulation training, and in many other aspects, such as biomechanical study. The Re-gong Tangka 3D data using motion capture technology can be used to build a database, but also can greatly improve the efficiency, reduce the cost of animation and animation, the animation production process more intuitive, more vivid effect. Figure 3 is based on human motion capture data flow chart of 3D character animation generation.

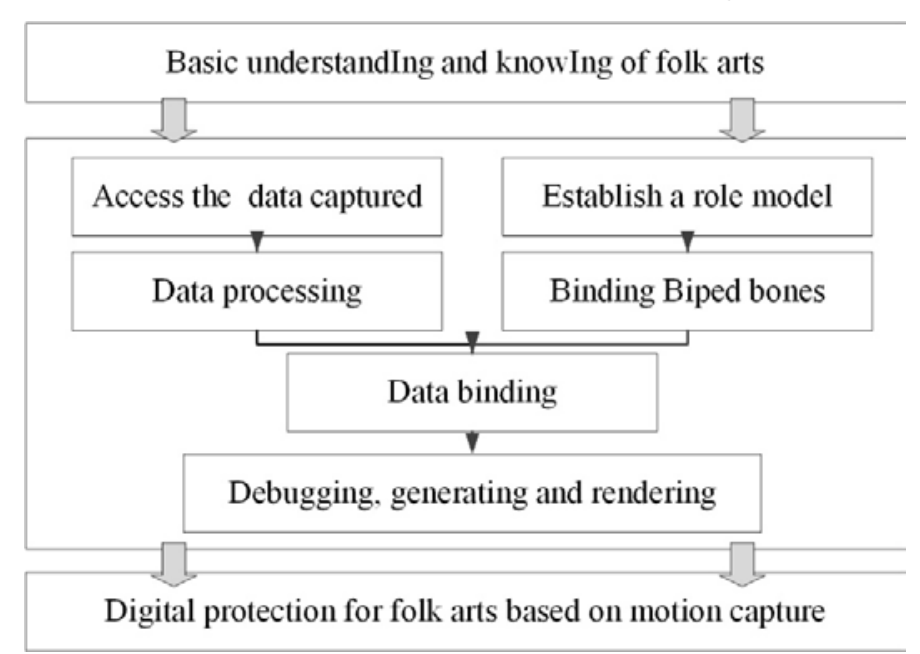

Figure 3.The digital protection technology based on motion capture

The 3D motion capture the Re-gong Tangka and skeletal binding motion data binding data based on the model, can generate character animation. Motion capture data will eventually need to model as a specific action vector display space performance, accurate motion capture data binding and appropriate model has obvious effect, the process of creating and role model itself is also very important. On the basis of the Re-gong Tangka as the background, this paper established a realistic character models and national characteristics. After the completion of the operation role model, you can use the Re-gong Tangka after 3D motion data binding framework role model driven, this process is called motion data binding.

\section{Value and Significance}

From the cultural aspect, national folk heritage artists Re-gong area died, single inheritance way cautious conservative plus, and young people affected by the impact of modern material civilization, have to work in the city, the inheritance of worrying. At the same time the Re-gong Art Resources loss. Rely on oral teaching that inspires true understanding within the way to inherit the cultural heritage is disappearing, traditional craftsmanship is on the verge of extinction. A large number of 
precious objects and materials with historical and cultural value of the abandoned or lost in foreign countries. At this time the use of modern multimedia means the protection and inheritance of Tibetan Buddhist art, the art of Tibetan Buddhism spread wider, spread faster, art reproduction by people all over the world whenever and wherever possible to appreciate; at the same time the digital protection of the Re-gong Tangka art, in digital museum, image database, website, virtual reality display, the realization of the network display sales of the Tibetan Buddhism art, games and other forms of mobile terminal. To have the inheritance and protection of traditional cultural heritage. The output of Chinese traditional culture to the world. This is consistent with the policy orientation of the revitalization of national culture. Culture is a nation's spirit and soul, is a decisive factor for national development and the development process, can profoundly affect a country. While carrying the digital dissemination of Buddhist culture in China Re-gong Tangka art, will to the Chinese culture to good effect in the global image establishment.

From the social significance, in today's society, many kinds of phenomena are and people's mentality imbalance. Our material civilization has developed to a certain extent, but the development of spiritual civilization and education has not kept pace, thus causing all kinds of discontent and resentment at present social mood. The Re-gong Tangka culture and carry forward the tradition can let the audience accepted and recognized its behind "the compassion is by this beautiful and stunning art form, to purify one heart" of the Buddhist cultural idea, enhance the realm of spiritual civilization in the present material society of man; so as to maintain social stability and ecological balance of the spirit. This is the current our country society material civilization to rise to a certain extent the most urgent demand. At the same time, can also to enhance the Tibetan tourism and economic benefits, promote the integration of Tibetan and Han people, maintaining the harmony and stability of the Tibetan.

By means of modern multimedia, Tibetan Buddhism Art would be preserved and inherited and spread much faster in a bigger scale. Thus, replicas can be shared and appreciated around the world. Meanwhile, the art would be on display and sell on the Internet through programs like Digital Museum, Image Database, VR Display and MT Games etc. The digitalized communication of Re-gong Thangka in a world scale will undoubtedly has a positive effect on the situation of Chinese culture in the globe.

In addition, people would accept the principle of Buddhism, namely, "Benevolence and clear your mind" through means talked just now. Further, the spirit of people living in a materialized society can be purified and improved, and social stability and spirit ecological balance can be kept.

\section{References}

[1] Lindsay MacDonald, 《Digital Heritage》 , Routledge Press 2008-4-8

[2] Mingquan Zhou, Guohua Geng and Zhongke Wu, 《Digital Preservation Technology for Cultural Heritage》, Springer Press, 2012-10-16

[3] By Filippo Stanco, Sebastiano Battiato, and Giovanni Gallo, 《Digital Imaging for Cultural Heritage Preservation: Analysis, Restoration, and Reconstruction of Ancient Artworks》 (Digital Imaging and Computer Vision), CRC Press, 2011-7-28

[4] Lindsay MacDonald, 《Digital Heritage》 , Routledge Press 2008-4-8

[5] Research on Modelling Digital Paper-cut Preservation, International Journal of Automation \& Computing,2009-11-15 